\title{
Prevalência de cárie em escolares de 5 e 12 anos no município de Palmas-TO
}

\author{
Prevalence of caries in school children of 5 and 12 years in the municipality of Palmas-TO \\ Revalencia de caries en niños de 5 y 12 años en el município de Palmas-TO
}

\author{
Veruska Azevedo Veras \\ ORCID: https://orcid.org/0000-0003-4949-2192 \\ Universidade Federal do Tocantins, Brasil \\ E-mail: veruska.veras@gmail.com \\ Eskálath Morganna Silva Ferreira \\ ORCID: https://orcid.org/0000-0002-2298-3656 \\ Universidade Federal do Tocantins, Brasil \\ E-mail: morganna_ferreira@hotmail.com \\ Leila Ruth Oliveira Gurgel do Amaral \\ ORCID: https://orcid.org/0000-0002-0863-4580 \\ Universidade Federal do Tocantins, Brasil \\ E-mail: leila.gurgel@uft.edu.br \\ Juliana Fonseca Moreira da Silva \\ ORCID: https://orcid.org/0000-0002-9200-0387 \\ Universidade Federal do Tocantins, Brasil \\ E-mail: julianafmsilva@mail.uft.edu.br
}

\begin{abstract}
Resumo
Objetivo: o objetivo desse estudo foi fazer uma análise da prevalência de cárie em crianças e adolescentes da região Norte e Palmas, capital do Tocantins. Metodologia: o método utilizado foi revisão integrativa da literatura, consistindo na busca de artigos científicos e dados oficiais de pesquisas nas bases de dados International library of medicine national institutes of health (PubMed), biblioteca virtual em saúde (BVS), Scientific Electronic Library Online (SCIELO) e Google acadêmico. Os dados são provenientes dos índices de cárie dentária (ceo-d - dentes decíduos cariados, perdidos por cárie e obturados e CPO-D - dentes permanentes cariados, perdidos por cárie e obturados) obtidos nos levantamentos epidemiológicos realizados no Brasil nos anos de 1986, 1996, 2003 e 2010. Resultados: embora os inquéritos epidemiológicos tenham registrado ao longo dos anos uma redução na prevalência da cárie dentária em países desenvolvidos e o Brasil segue essa tendência mundial, a cárie ainda é considerada um problema de saúde pública. $\mathrm{O}$ estudo demonstrou que o índice de cárie dentária em Palmas é maior em crianças do que em adolescentes. O percentual de dentes restaurados, componente "o" do índice ceo-d, é menor nas crianças de cinco anos de idade da capital. O estudo evidenciou que a cárie afeta desproporcionalmente a população menos favorecida, permanecendo ao longo dos anos diferenças significativas na prevalência de cárie entre as regiões do país. Poucos dados foram encontrados na literatura referentes ao Estado do Tocantins e Palmas. Conclusão: conclui-se que ações de promoção e prevenção em saúde bucal no município de Palmas deverão ser implementadas em idades iniciais de forma efetiva com o objetivo de reduzir a prevalência de cárie na infância.
\end{abstract}

Palavras-chave: Cárie dental; Epidemiologia; Prevalência; Índice CPO.

\begin{abstract}
Objective: the objective of this study was to analyze the prevalence of children and adolescents in the North region and Palmas, capital of Tocantins. Methodology: the method used was the integrative literature review, consisting of the search for scientific and official articles from databases International library of Medicine National Institutes of Health (PubMed), virtual health library (VHL research), Scientific Electronic Library Online (SCIELO) and Google Axel. Data are derived from dental caries indices ( $\mathrm{dmft}$ - decayed deciduous teeth, lost due to caries and filled and DMFT permanent decayed teeth, lost to decay and filled) obtained in epidemiological surveys carried out in Brazil in 1986, 1996, 2003 and 2010. Results: although epidemiological surveys have registered a reduction in the prevalence of dental caries in developed countries over the years and Brazil follows this global trend, childhood is still a public health problem. The study that the rate of dental caries in Palmas is higher in children than in adolescents. The percentage of restorative teeth, component "o" of the dmft index, is lower in five-year-old children in the capital. The study showed that tooth decay disproportionately affects the underprivileged population, remaining over the years marked differences in the prevalence of caries between and across the country. Few data were found in the literature referring to the State of Tocantins and Palmas. Conclusion: it is concluded that oral health promotion and prevention actions were prevented until unintentional childhood at early ages in a way with the municipality of prevalence in oral age.
\end{abstract}

Keywords: Dental caries; Epidemiology; Prevalence; DMF index. 


\begin{abstract}
Resumen
Objetivo: el objetivo de este estudio fue analizar la prevalencia de caries en niños y adolescentes de la región Norte y Palmas, capital de Tocantins. Metodología: el método utilizado fue una revisión integrativa de la literatura, consistente en la búsqueda de artículos científicos y datos oficiales de investigación en las bases de datos de la Biblioteca Internacional de Medicina de los Institutos Nacionales de la Salud (PubMed), Biblioteca Virtual en Salud (BVS), Scientific Electronic Library Online. SCIELO) y Google Scholar. Los datos provienen de los índices de caries dental (dmft - dientes temporales cariados, perdidos por caries y obturados y CPOD - dientes permanentes cariados, perdidos por caries y obturados) obtenidos en encuestas epidemiológicas realizadas en Brasil en 1986, 1996, 2003 y 2010 Resultados: aunque las encuestas epidemiológicas han registrado una reducción en la prevalencia de caries dental en los países desarrollados a lo largo de los años y Brasil sigue esta tendencia mundial, la caries todavía se considera un problema de salud pública. El estudio mostró que la tasa de caries dental en Palmas es mayor en niños que en adolescentes. El porcentaje de dientes restaurados, componente "o" del índice dmft, es menor en los niños de cinco años de la capital. El estudio mostró que la caries afecta de manera desproporcionada a la población desfavorecida, manteniéndose diferencias significativas en la prevalencia de caries entre las regiones del país a lo largo de los años. Pocos datos fueron encontrados en la literatura referentes al Estado de Tocantins y Palmas. Conclusión: se concluye que las acciones de promoción y prevención de la salud bucal en la ciudad de Palmas deben implementarse de manera efectiva en edades tempranas para disminuir la prevalencia de caries infantil.
\end{abstract}

Palabras clave: Caries dental; Epidemiología; Prevalencia; Índice CPO.

\title{
1. Introdução
}

Embora seja evitável, a cárie ainda é considerada um importante problema de saúde pública, pois apesar da redução da carga da doença em crianças nas últimas décadas, a prevalência ainda é alta, sendo que em alguns países ultrapassa a 50\% em crianças de 12 anos (Silva et al., 2019; Xiao et al., 2019; Freire et al., 2013; Splieth, et al., 2019). A doença afeta desproporcionalmente a população mais desfavorecida e cada vez mais o mundo se torna dividido e polarizado com grandes diferenças econômicas e sociais, as quais afetam diretamente a saúde da população (Watt et al., 2018).

Vários fatores de risco estão associados ao desenvolvimento da cárie em crianças como fatores comportamentais de higiene oral e alimentares, experiência previa de cárie, fatores biológicos e fatores socioeconômicos (Kopycka-Kedzierawski, \& Billings, 2011).

Na maioria dos países desenvolvidos houve um declínio na prevalência de cárie dentária nas três últimas décadas do século XX e início do século XXI, apesar de ainda permanecer elevada na população com menor renda, em determinadas regiões e cidades do interior e entre diferentes grupos populacionais (Narvai et al., 2006; Brasil, 2012).

O Brasil tem acompanhado a tendência mundial na queda da prevalência de cárie em escolares, apesar de ter apresentado historicamente elevados índices de cárie dentária ao longo dos anos (Vilar et al., 2020). Mesmo com a melhora dos índices de saúde bucal na população, a cárie ainda é uma das doenças bucais que mais acomete os brasileiros, principalmente crianças, adultos e idosos (Agnelli, 2015; Dourado et al., 2017; Peres et.al., 2012).

Inquéritos epidemiológicos em saúde bucal são elementos fundamentais no conhecimento da prevalência da cárie dentária, nos processos de monitoramento das condições de saúde da população e no desempenho do sistema de saúde (Peres et.al., 2012; Roncalli et al., 2019). Pontigo-Loyola et al. (2020) afirmam que os estudos epidemiológicos são considerados de extrema importância para a saúde pública por constituírem a única fonte de informações precisas sobre a frequência e distribuição das doenças bucais, servindo de base para a avaliação das necessidades de tratamento dentário.

Embora o Ministério da Saúde, em 1980, baseado em levantamentos parciais realizados no território brasileiro, tenha obtido valor para o índice CPO-D igual a 7,3, indicando uma prevalência "muito alta" para cárie dentária no Brasil, são considerados oficialmente quatro levantamentos epidemiológicos em saúde bucal a nível nacional, em 1986, 1996, 2003 e 2010 (Narvai et al., 2006; Agnelli, 2015; Ardenghi et al., 2013; Tobias et al., 2008)

A organização mundial da saúde - OMS preconiza o instrumento Índice CPO-D, formulado por Klein e Palmer, em 1937, para avaliar a prevalência da cárie dentária, o qual tem origem nas palavras “cariados", "perdidos" e "obturados", e o "D" 
indica que a unidade de medida que é o dente. A OMS preconiza os valores do índice conforme ao grau de severidade, podendo ser considerado muito baixo ( 0,0 a 1,1), baixo (1,2 a 2,6), moderado (2,7 a 4,4), alto (4,5 a 6,5) e muito alto (6,6 e mais) (Dourado et al., 2017; Moura et al., 2008)

Poucos experimentos de levantamento epidemiológico em saúde bucal foram realizados no Brasil, enquanto vários países detêm bases de dados de cárie dentária desde as primeiras décadas (Melo \& Lima, 2009). Dentre os estudos encontrados na literatura a maioria foi realizado em capitais ou grandes cidades, sendo observado poucos dados referentes a municípios pequenos e população rural (Dourado et al., 2017).

As dimensões continentais e as diferenças socioeconômicas entre as regiões do país devem ser consideradas ao analisarmos a prevalência de cárie no Brasil, pois os últimos levantamentos têm demonstrado que há grandes discrepâncias no índice de cárie por regiões, como no estudo de 2010 que registrou uma diferença de $84 \%$ entre as regiões com valores extremos, Norte $(3,16)$ e Sudeste $(1,72)$ (Brasil, 2012).

Considerando a importância de avaliar as condições de saúde bucal da população de escolares de 5 e 12 anos do município de Palmas, o presente estudo tem como objetivo realizar revisão da literatura dos índices de cárie dentária na Região Norte, estado do Tocantins e sua capital Palmas, analisando os fatores que contribuíram para a redução da prevalência, bem como as necessidades e conquistas a serem enfrentadas no futuro.

\section{Metodologia}

Foi realizado um estudo descritivo por meio de uma revisão integrativa da literatura, através da busca de artigos científicos e dados oficiais de pesquisas nas bases de dados, International library of medicine national institutes of health (PubMed), biblioteca virtual em saúde (BVS), Scientific Electronic Library Online (SCIELO) e Google acadêmico, a partir das palavras-chaves: (tw:(prevalence)) AND (tw:(DMF index)) AND (tw:(EPIDEMIOLOGY)) AND (tw:(dental carie)), cadastradas nos Descritores em Ciências da Saúde (Decs). A coleta de dados ocorreu entre os anos de 1980 a 2021. As perguntas norteadoras da pesquisa foram: Qual o índice de cárie nos escolares no município de Palmas? Qual a situação de saúde bucal das crianças? As crianças do município apresentam um índice de cárie maior comparadas às demais capitais?

A busca foi realizada em língua portuguesa e inglesa. A pré-seleção dos artigos foi realizada inicialmente através da leitura dos títulos e resumos, e posteriormente foi realizada uma leitura minuciosa dos artigos selecionados, seguindo os critérios de seleção previamente estabelecidos como: serem artigos com acesso online aberto, artigos originais nos idiomas português e inglês, artigos que respondem às questões norteadoras da pesquisa, e estudos que utilizaram como instrumento de medida o Índice CPO-D (dentição permanente) e ceo-d (dentição decídua) para avaliar a prevalência de cárie dentária nas faixas etárias de 5 e 12 anos. Para a definição dos critérios de exclusão considerou-se estudos fora dos instrumentos de medida descritos, fuga do tema proposto e artigos não disponíveis por completo.

\section{Resultados e Discussão}

Por meio das bases de dados descritas foram obtidos 186 artigos através dos critérios de pesquisa aplicados. No entanto, após a leitura dos títulos e resumos foram excluídos 112 estudos que não se apresentaram de acordo com a temática da pergunta norteadora da pesquisa, totalizando 60 artigos na triagem inicial. Estes, porém, foram lidos na integra observando os critérios de inclusão e exclusão determinados pela pesquisa, onde 36 foram excluídos por não cumprirem com a proposta do trabalho. Ao final 24 artigos foram selecionados de acordo com os critérios estabelecidos, ao qual foram incluídos nesse estudo por serem relevantes para a revisão integrativa. A descrição detalhada do processo de seleção dos artigos encontra-se demonstrado na Figura 1. 
Figura 1. Fluxograma das etapas de seleção e identificação dos artigos selecionados.
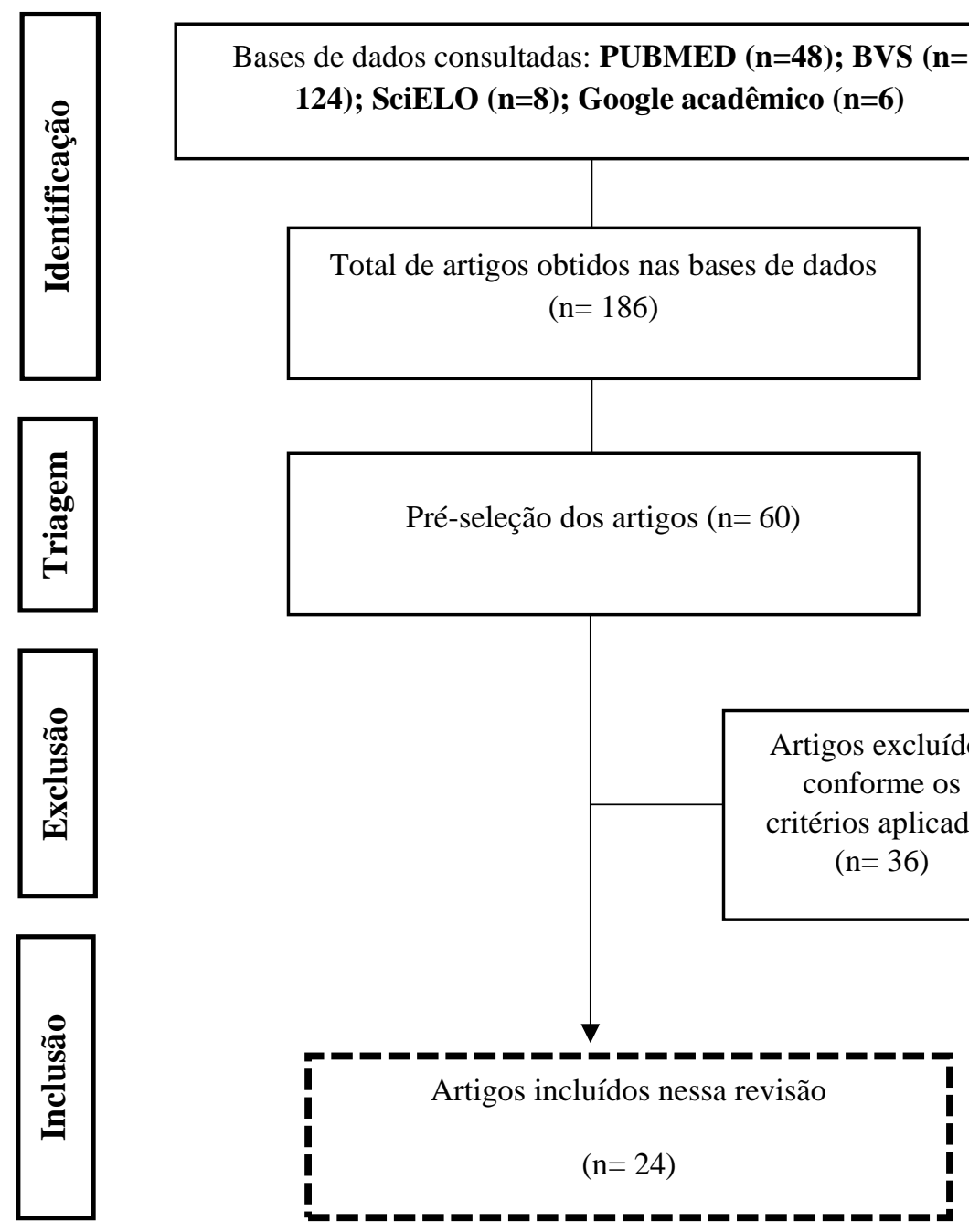

124); SciELO (n=8); Google acadêmico $(n=6)$

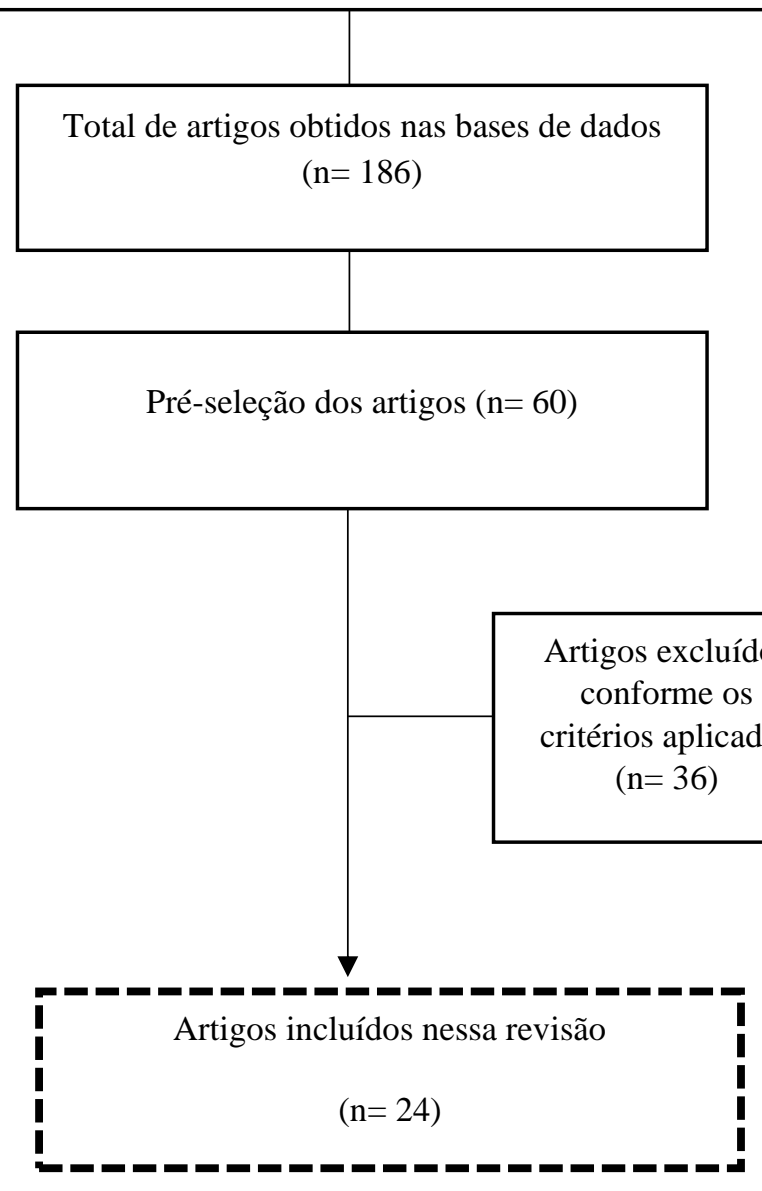

Fonte: Autores.

A partir dos 24 artigos selecionados através da coleta de dados foi elaborada uma análise como o objetivo de promover uma síntese dos estudos elegidos. Esta foi direcionada a partir da construção de um quadro (Quadro 1), sendo este, organizado por autores e ano de publicação, objetivos da pesquisa, tipo do estudo, bem como resultados ou considerações de cada estudo.

Quadro 1. Síntese dos artigos selecionados para a revisão integrativa, apresentados pelos autores e ano de publicação, objetivo do estudo, tipo de estudos empregado, faixa etária dos pacientes, local de estudo e resultados obtidos.

\begin{tabular}{|c|c|c|c|c|c|}
\hline Autores (ano) & Objetivo & Tipo de estudo & Faixa etária & $\begin{array}{c}\text { Local do } \\
\text { estudo }\end{array}$ & Resultados ou Conclusões \\
\hline $\begin{array}{c}\text { Antunes \& } \\
\text { Narvai, } 2010\end{array}$ & $\begin{array}{l}\text { Sistematizar o conhecimento } \\
\text { disponível quanto ao estágio atual de } \\
\text { efetivação dessas medidas e seu } \\
\text { impacto sobre as desigualdades em } \\
\text { saúde }\end{array}$ & $\begin{array}{l}\text { Revisão de } \\
\text { literatura }\end{array}$ & & Brasil & $\begin{array}{l}\text { A fluoretação da água de abastecimento público é } \\
\text { claramente uma estratégia de intervenção sobre os } \\
\text { determinantes populacionais da cárie dentária, } \\
\text { constituindo um dos elementos mais importantes da } \\
\text { redução dos indicadores da doença no País e no } \\
\text { exterior. }\end{array}$ \\
\hline Agnelli, 2015 & $\begin{array}{l}\text { Analisar os valores de CPOD } \\
\text { registrados no Brasil, de } 1980 \text { a } 2010 \text {, e } \\
\text { os fatores que influenciaram a } \\
\text { prevalência de cárie no país e, } \\
\text { consequentemente, a alteração do } \\
\text { índice, neste período. }\end{array}$ & $\begin{array}{l}\text { Revisão de } \\
\text { literatura }\end{array}$ & 12 anos & Brasil & $\begin{array}{l}\text { A situação da prevalência de cárie na população } \\
\text { brasileira, no período considerado, foi de declínio e } \\
\text { polarização. Em nível nacional, a prevalência } \\
\text { diminuiu, mas a doença permaneceu mais comum na } \\
\text { população menos favorecida economicamente. }\end{array}$ \\
\hline $\begin{array}{l}\text { Ardenghi et al., } \\
2013\end{array}$ & $\begin{array}{l}\text { Avaliar a influência de desigualdades } \\
\text { sociais de ordem individual e } \\
\text { contextual na experiência de cárie } \\
\text { dentária não tratada em crianças no } \\
\text { Brasil. }\end{array}$ & $\begin{array}{l}\text { Estudo } \\
\text { transversal }\end{array}$ & $\begin{array}{c}\text { anos, } 12 \\
\text { anos, } 15-19 \\
\text { anos, } 35-44 \\
\text { anos e } 50-59 \\
\text { anos }\end{array}$ & Brasil & $\begin{array}{l}\text { Desigualdades na prevalência de cárie não tratada } \\
\text { persistem, afetando as crianças com dentição } \\
\text { decídua no Brasil. O planejamento de medidas } \\
\text { públicas para a promoção de saúde bucal deve }\end{array}$ \\
\hline
\end{tabular}


Research, Society and Development, v. 11, n. 2, e31211225667, 2022

(CC BY 4.0) | ISSN 2525-3409 | DOI: http://dx.doi.org/10.33448/rsd-v11i2.25667

\begin{tabular}{|c|c|c|c|c|c|}
\hline & & & & & $\begin{array}{l}\text { considerar o efeito de fatores contextuais como } \\
\text { determinante de riscos individuais. }\end{array}$ \\
\hline Brasil, 2004 & $\begin{array}{c}\text { Pesquisa nacional sobre índice de cárie } \\
\text { na população }\end{array}$ & $\begin{array}{l}\text { Estudo transver } \\
\text { sal de base } \\
\text { populacional }\end{array}$ & $\begin{array}{c}18 \text { a } 36 \\
\text { meses, } 5 \text { anos, } \\
12 \text { anos, } 15- \\
19 \text { anos, } 35- \\
44 \text { anos e } 50- \\
59 \text { anos }\end{array}$ & $\begin{array}{l}250 \text { municípios } \\
\text { distribuídos em } \\
\text { portes } \\
\text { populacionais, } \\
\text { além da área } \\
\text { rural, incluindo } \\
50 \text { cidades da } \\
\text { região } \\
\text { amazônica } \\
\end{array}$ & $\begin{array}{l}\text { A análise dos dados gerados na pesquisa retrata o } \\
\text { perfil de saúde bucal da população. }\end{array}$ \\
\hline Brasil, 2012 & $\begin{array}{c}\text { Pesquisa nacional sobre índice de cárie } \\
\text { na população }\end{array}$ & $\begin{array}{l}\text { Estudo transver } \\
\text { sal de base } \\
\text { populacional }\end{array}$ & $\begin{array}{c}5 \text { anos, } 12 \\
\text { anos, } 15-19 \\
\text { anos, } 35-44 \\
\text { anos e } 50-59 \\
\text { anos }\end{array}$ & $\begin{array}{c}26 \text { capitais e } \\
\text { Distrito Federal, } \\
\text { envolvendo as } \\
\text { cinco regiões, } \\
\text { com } \\
\text { participação de } \\
177 \text { municípios } \\
\end{array}$ & $\begin{array}{l}\text { Os resultados do Projeto SB Brasil } 2010 \text { indicam } \\
\text { que, segundo a classificação adotada pela OMS, o } \\
\text { Brasil saiu de uma condição de média prevalência } \\
\text { de cárie em } 2003 \text { (CPO entre } 2,7 \text { e } 4,4 \text { ), para uma } \\
\text { condição de baixa prevalência em } 2010 \text { (CPO entre } \\
1,2 \text { e 2,6). }\end{array}$ \\
\hline Berti et al., 2013 & $\begin{array}{c}\text { estimar, para a população de escolares } \\
\text { de } 5 \text { e } 12 \text { anos do município de } \\
\text { Cascavel, a prevalência de cárie } \\
\text { dentária, }\end{array}$ & $\begin{array}{l}\text { estudo } \\
\text { transversal. }\end{array}$ & 5 e 12 anos & $\begin{array}{l}\text { Cascavel, } \\
\text { Paraná }\end{array}$ & $\begin{array}{l}\text { Com relação ao ceo-d avaliado em escolares de } 5 \\
\text { anos de idade foi observado um comportamento } \\
\text { semelhante ao CPO-D aos } 12 \text { anos. As regiões que } \\
\text { apresentaram índices maiores foi centro e norte. }\end{array}$ \\
\hline Borges et al., 2016 & $\begin{array}{l}\text { Descrever os fatores associados à cárie } \\
\text { dentária em escolares }\end{array}$ & $\begin{array}{l}\text { Estudo } \\
\text { transversal }\end{array}$ & 10 a 17 anos & $\begin{array}{l}\text { Santa Cruz do } \\
\text { Sul, Rio Grande } \\
\text { do Sul }\end{array}$ & $\begin{array}{l}\text { A presença de cárie em alunos no sul do Brasil foi } \\
\text { associada com residência em áreas rurais, mãe com } \\
\text { baixo nível educacional e frequentar escola pública }\end{array}$ \\
\hline $\begin{array}{l}\text { Cruz \& Narvai, } \\
\quad 2018\end{array}$ & $\begin{array}{l}\text { Analisar a associação entre a } \\
\text { exposição à água fluoretada e cárie } \\
\text { dentária, em contexto de uso } \\
\text { generalizado de creme dental } \\
\text { fluoretado no Brasil, em cenário de } \\
\text { baixa prevalência da doença. }\end{array}$ & $\begin{array}{l}\text { Estudo } \\
\text { observacional } \\
\text { transversal, de } \\
\text { tipo censitário }\end{array}$ & $\begin{array}{l}\text { Adolescentes } \\
\text { de } 11 \text { e } 12 \\
\text { anos, }\end{array}$ & $\begin{array}{l}\text { Silveiras e São } \\
\text { José do Barreiro } \\
\text { (Vale do } \\
\text { Paraíba) }\end{array}$ & $\begin{array}{l}\text { O valor do índice CPO-D foi menor no município } \\
\text { com exposição ao flúor na rede de abastecimento, } \\
\text { ainda que ambas as populações tenham estado } \\
\text { expostas aos fluoretos veiculados pelos cremes } \\
\text { dentais nesses municípios }\end{array}$ \\
\hline $\begin{array}{l}\text { Gimenez et al., } \\
2016\end{array}$ & $\begin{array}{l}\text { Analisar a prevalência de cárie em } \\
\text { crianças da América Latina e Caribe } \\
\text { considerando estudos realizados neste } \\
\text { novo século. }\end{array}$ & $\begin{array}{l}\text { revisão } \\
\text { sistemática com } \\
\text { meta-análise }\end{array}$ & $\begin{array}{l}\text { Crianças de } \\
5-6 \text { ou } 11-13 \\
\text { anos }\end{array}$ & $\begin{array}{l}\text { Literatura } \\
\text { científica da } \\
\text { região da } \\
\text { América Latina } \\
\text { e Caribe. } \\
\end{array}$ & $\begin{array}{l}\text { Apesar do declínio da prevalência de cárie em } \\
\text { dentes permanentes, principalmente no Brasil, a } \\
\text { doença ainda atinge mais da metade da população } \\
\text { infantil em países da América Latina e Caribe no } \\
\text { século XXI. }\end{array}$ \\
\hline Huang et al., 2019 & $\begin{array}{l}\text { Apresenta dados básicos de nutrição e } \\
\text { saúde bucal em uma amostra de } \\
\text { conveniência de crianças de } 2 \text { a } 5 \text { anos } \\
\text { e suas mães/cuidadores que } \\
\text { participaram de um estudo preventivo } \\
\text { baseado em escola projetado para } \\
\text { entender a nutrição infantil e a saúde } \\
\text { bucal. }\end{array}$ & $\begin{array}{l}\text { Estudo } \\
\text { descritivo }\end{array}$ & $\begin{array}{l}\text { Crianças de } 2 \\
\text { a } 5 \text { anos e } \\
\text { suas mães }\end{array}$ & Vietnã & $\begin{array}{l}\text { A maioria das mães e crianças sofria de doenças } \\
\text { dentárias não tratadas. Os programas de saúde } \\
\text { pública devem se concentrar na nutrição e na } \\
\text { promoção da saúde bucal, bem como no tratamento } \\
\text { odontológico desde a gravidez e o nascimento. }\end{array}$ \\
\hline $\begin{array}{l}\text { Kopycka- } \\
\text { Kedzierawski et } \\
\text { al., } 2011\end{array}$ & $\begin{array}{l}\text { Avaliar a prevalência de cárie dentária } \\
\mathrm{e} \text { a utilização de atendimento } \\
\text { odontológico em crianças pré-escolares }\end{array}$ & $\begin{array}{l}\text { Ensaio clinico } \\
\text { randomizado }\end{array}$ & $\begin{array}{c}234 \text { crianças } \\
\text { entre } 12 \text { a } 60 \\
\text { meses } \\
\text { matriculadas } \\
\text { em creches } \\
\text { urbanas } \\
\end{array}$ & $\begin{array}{l}\text { Rochester, } \\
\text { Nova York }\end{array}$ & $\begin{array}{l}\text { Os dados mostram que } 28 \% \text { das crianças tinham } \\
\text { cárie e, destas, } 61 \% \text { nunca haviam sido tratadas de } \\
\text { cárie, indicando que são necessários esforços } \\
\text { contínuos para melhorar a utilização dos cuidados } \\
\text { de saúde bucal por crianças pré-escolares do centro } \\
\text { da cidade. }\end{array}$ \\
\hline $\begin{array}{l}\text { Lacerda et al., } \\
\quad 2020\end{array}$ & $\begin{array}{l}\text { Levantar dados sobre a existência do } \\
\text { heterocontrole nos } 10 \text { maiores } \\
\text { municípios do estado do Tocantins, } \\
\text { analisar a concentração de fluoreto } \\
\text { presente na água de abastecimento } \\
\text { público destas cidades }\end{array}$ & $\begin{array}{c}\text { Pesquisa } \\
\text { transversal de } \\
\text { campo }\end{array}$ & & $\begin{array}{c}\text { Palmas, } \\
\text { Araguaína, } \\
\text { Gurupi, Porto } \\
\text { Nacional, } \\
\text { Paraiso do } \\
\text { Tocantins, } \\
\text { Araguatins, } \\
\text { Colinas, Guaraí, } \\
\text { Tocantinópolis } \\
\text { e } \\
\text { Dianópolis.(Toc } \\
\text { antins) }\end{array}$ & $\begin{array}{l}80,2 \% \text { das amostras analisadas não estavam } \\
\text { adequadas no que diz respeito a quantidade de } \\
\text { fluoretos, onde } 8 \text { municípios possuem flúor } \\
\text { agregado a água, porém, } 9 \text { em cada } 10 \text { amostras não } \\
\text { estão no padrão adequado de menor risco a fluorose } \\
\text { dentária e maior proteção contra a cárie dentária }\end{array}$ \\
\hline Lucas et al., 2005 & $\begin{array}{l}\text { Analisar o cumprimento de metas da } \\
\text { OMS para a cárie dentária no ano } 2000 \\
\text { em Minas Gerais }\end{array}$ & $\begin{array}{l}\text { Estudo } \\
\text { ecológico }\end{array}$ & 5 e 12 anos & $\begin{array}{l}\text { Municípios do } \\
\text { Estado de } \\
\text { Minas Gerais. }\end{array}$ & $\begin{array}{l}\text { Os resultados não devem ser generalizados, mas } \\
\text { indicam iniqüidades na saúde bucal e o papel } \\
\text { desempenhado por fatores sócio-econômicos e a } \\
\text { fluoretação da água. }\end{array}$ \\
\hline $\begin{array}{l}\text { Martins et al., } \\
\quad 2005\end{array}$ & $\begin{array}{l}\text { Descrever } \\
\text { os principais aspectos dos quatro } \\
\text { levantamentos } \\
\text { epidemiológicos de base populacional } \\
\text { brasileira }\end{array}$ & $\begin{array}{l}\text { Revisão de } \\
\text { literatura }\end{array}$ & & Brasil & 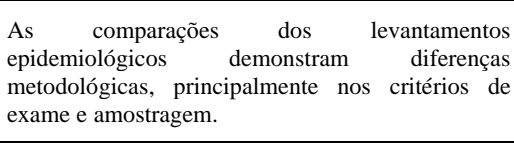 \\
\hline $\begin{array}{l}\text { Melo \& Lima, } \\
\quad 2009 .\end{array}$ & $\begin{array}{l}\text { Analisar o perfil epidemiológico da } \\
\text { cárie dentária no Brasil no período de } \\
1986 \text { a } 2003\end{array}$ & $\begin{array}{l}\text { Pesquisa } \\
\text { bibliográfica } \\
\text { em registros } \\
\text { estatísticos e } \\
\text { acervos } \\
\text { documentais }\end{array}$ & $\begin{array}{c}5 \text { anos, } 12 \\
\text { anos, } 15-19 \\
\text { anos, } 35-44 \\
\text { anos e } 50-59 \\
\text { anos }\end{array}$ & Brasil & $\begin{array}{l}\text { Ocorreu declínio da cárie dentária em várias regiões } \\
\text { brasileiras, evidenciado pelos dados nacionais, pelo } \\
\text { aumento da oferta de dentifrícios fluoretados, } \\
\text { reorganização da prática odontológica, dentre outros } \\
\text { fatores. }\end{array}$ \\
\hline $\begin{array}{l}\text { Moysés et al., } \\
2013\end{array}$ & $\begin{array}{l}\text { Abordar o conceito de vigilância à } \\
\text { saúde aplicado para a área da saúde } \\
\text { bucal com base nos dados do inquérito } \\
\text { epidemiológico nacional em saúde } \\
\text { bucal - o projeto SBBrasil } 2010 .\end{array}$ & $\begin{array}{l}\text { Revisão crítica } \\
\text { e integrativa }\end{array}$ & & Brasil & $\begin{array}{l}\text { A concretização do SB Brasil } 2010 \text { evidenciou que } \\
\text { é possível construir política e operacionalmente } \\
\text { maior integração entre a academia e os serviços de } \\
\text { saúde. }\end{array}$ \\
\hline Narvai et al., 1999 & $\begin{array}{l}\text { Analisar alguns dados nacionais sobre } \\
\text { cárie dentária em dentes permanentes } \\
\text { de escolares brasileiros nas últimas } \\
\text { décadas do século XX. }\end{array}$ & $\begin{array}{l}\text { Revisão de } \\
\text { literatura }\end{array}$ & $\begin{array}{c}5 \text { anos, } 12 \\
\text { anos, } 15-19 \\
\text { anos, } 35-44\end{array}$ & Brasil & $\begin{array}{l}\text { O estudo concluiu que entre } 1980-1996 \text { houve um } \\
\text { ganho médio de cerca de } 4 \text { dentes sem experiência } \\
\text { de cárie na idade-índice de } 12 \text { anos. No entanto, tal }\end{array}$ \\
\hline
\end{tabular}




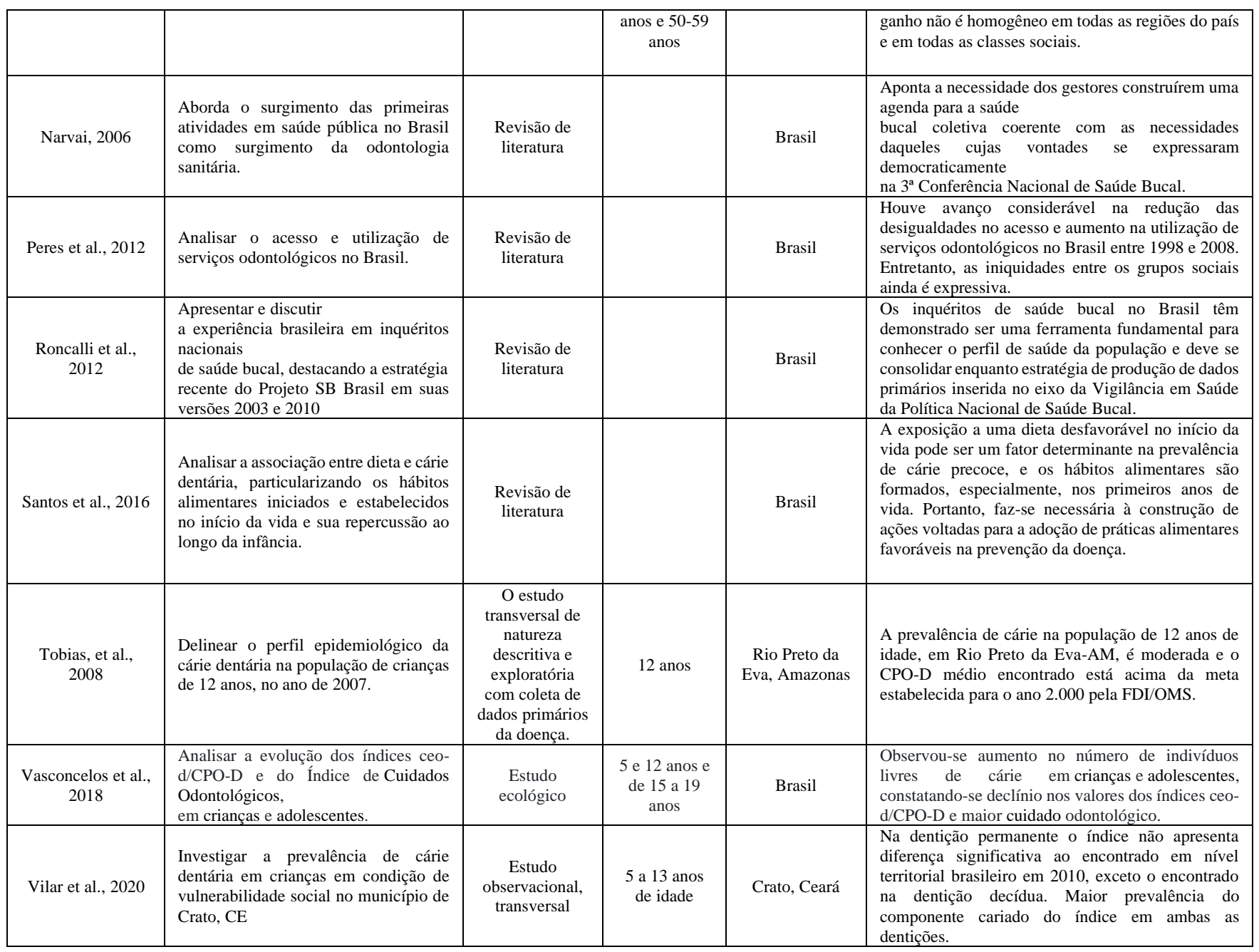

Fonte: Autores.

O primeiro levantamento epidemiológico no Brasil a nível nacional foi realizado em 1986 abrangendo a zona urbana de 16 capitais brasileiras, as quais representaram as cinco regiões no país. Foram pesquisados dados sobre cárie dentária, doença periodontal e necessidade de prótese, nas faixas etárias de 6-12 anos, 15-19 anos, 35-44 anos e 50-59 anos (Brasil, 2004). Vale ressaltar que o Estado do Tocantins não foi incluído neste estudo, pois teve sua criação em 1988.

O segundo estudo foi realizado em 1996 nas 27 capitais brasileiras, contando com Distrito Federal, (1.120 cidades) na população de 6 a 12 anos, onde foi avaliado somente a presença de cárie dentária nas crianças, demonstrando limitações para aferir situações regionais (Agnelli, 2015; Brasil, 2004; Martins et al., 2005).

Os últimos levantamentos, 2003 e 2010, foram os mais abrangentes pois seguiram as diretrizes padronizadas da OMS com acréscimos de dados socioeconômicos, auto percepção em saúde bucal, acesso aos serviços odontológicos e satisfação da população adulta e idosa. O levantamento epidemiológico de 2013, conhecido por "Projeto SB Brasil 2003", envolveu 250 municípios distribuídos em portes populacionais, além da área rural, incluindo 50 cidades da região amazônica. Foram levantados dados de cárie dentária, fluorose, doença periodontal, oclusopatias e edentulismo (Brasil, 2012; Agnelli, 2015; Roncalli et al., 2012).

O "Projeto SB BRASIL 2003”, mesmo considerando as diversas dificuldades operacionais, representou um expressivo avanço nos estudos epidemiológicos em saúde bucal, contribuindo, assim, com a consolidação da Vigilância em Saúde Bucal no âmbito do Sistema de Saúde Pública (SUS) (Roncalli et al., 2019). 
O "Projeto SB BRASIL 2010", foi realizado em 26 capitais e Distrito Federal, envolvendo as cinco regiões, com participação de 177 municípios, com 38 mil pessoas entrevistadas e examinadas (Brasil, 2012; Agnelli, 2015; Roncalli et al., 2019).

Embora os levantamentos epidemiológicos realizados pelo Ministério da Saúde no Brasil tenham produzidos estimativas consideradas válidas para os valores do índice de cárie dentária, a heterogeneidade metodológica entre eles é nítida e demonstra variações em relação à coleta de dados, ao tipo de investigação, delineamento da pesquisa, plano amostral, critério de diagnóstico para a doença e grande concentração de esforços pontuais (Narvai et al., 2006; Martins et al., 2005; Moyses et al., 2013).

O Brasil saiu de uma prevalência "muito alta" para cárie dentária com índice CPO-D para a idade-índice de 12 anos de 7,3 em 1980 para "baixa" prevalência em 2010 com valor de 2,1, assumindo a segunda classificação adotada pela OMS (Narvai et al., 2006).

Antunes e Narvai (2010) associaram a redução na prevalência de cárie a duas grandes intervenções fortemente expandidas no país, mesmo que de forma desigual, que tiveram grande importância nas duas últimas décadas: a fluoretação da água de abastecimento público e o atendimento odontológico na rede pública do Sistema Único de Saúde (SUS). O fortalecimento dessas estratégias de prevenção e promoção são de extrema importância a fim de reduzir as disparidades entre as regiões e comunidades de diferentes classes sociais (Agnelli, 2015).

Embora a OMS tenha estabelecido metas para a saúde bucal, os levantamentos epidemiológicos realizados em 1986 e 1996 não incluíram na amostra a idade de cinco anos, e sim de seis anos para as crianças. De acordo com Narvai et al. (1999) isso impossibilita avaliar a evolução da prevalência de cárie na faixa etária de 5 anos de idade como nos demais estudos. Narvai et al. (2006) afirmam ainda que o relatório técnico do levantamento epidemiológico realizado no ano de 1996 não se encontra disponível na literatura, pois o MS não publicou o relatório final da pesquisa.

O Brasil em 1986, segundo dados internacionais da época, assumiu o terceiro pior índice do mundo (CPO-D 6,7) com prevalência "muito alta" para cárie, ficando atrás somente de Brunei, República Dominicana e empatado com a Jamaica. Dez anos depois assume uma prevalência "média" com valor CPO-D de 3,1, ficando próximo da meta estabelecida pela OMS para o ano de 2000 que era CPO-D igual ou menor que 3 (Melo \& Lima, 2009). Na pesquisa de 2003, a classificação de prevalência "média" é mantida com valor de CPO-D igual a 2,8 e somente em 2010 o país atingiu uma "baixa" prevalência de cárie com valor CPO-D de 2,1 (Narvai et al., 2006; Agnelli, 2015; Ardenghi et al., 2013).

O Gráfico 1 demonstra a evolução do índice CPO-D para a idade-índice de 12 anos no Brasil e para cada uma das cinco regiões brasileiras nos levantamentos realizados em 1986, 1996, 2003 e 2010. Observa-se que ao longo dos anos houve declínio da prevalência de cárie em todas as regiões nos quatro levantamentos. No entanto as diferenças entre as regiões Norte, Nordeste e Centro-Oeste com as regiões Sul e Sudeste tendem a permanecer por décadas. Alguns autores afirmam que no Brasil a prevalência da cárie dentária entrou em declínio e polarização, ou seja, apesar da melhora nos índices, a distribuição da cárie ainda é desigual, onde cerca de 20\% da população concentra 60\% da doença (Vilar et al., 2020; Agnelli, 2015; Cruz \& Narvai, 2018). 
Gráfico 1. Evolução do índice CPO-D para 12 anos por região no Brasil.

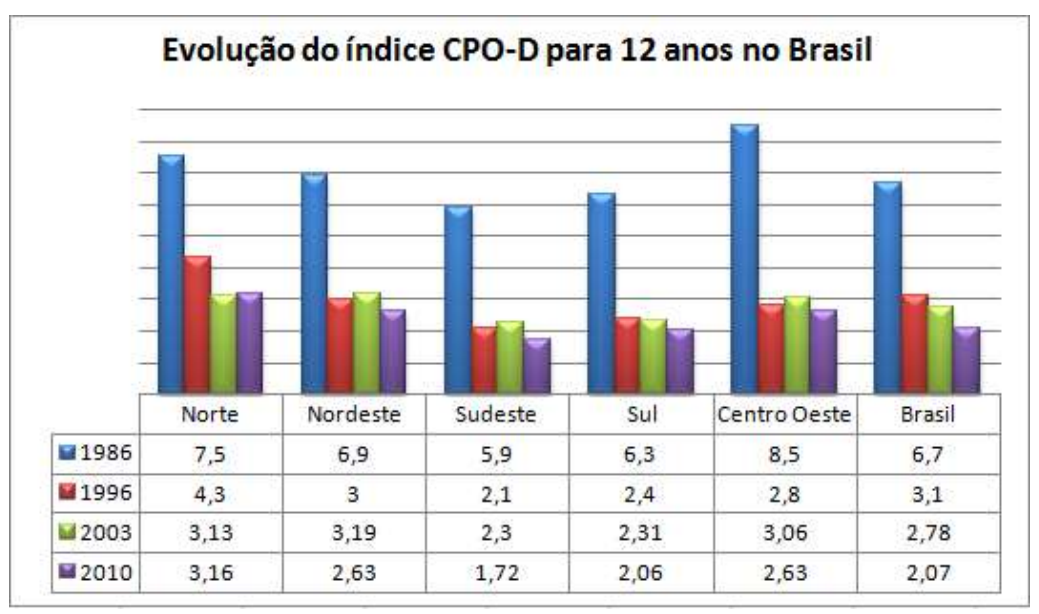

Fonte: Brasil (2004); Brasil (2012).

Comparando os estudos de 2003 e 2010, observa-se que das cinco regiões brasileiras, em quatro houve redução do índice COP-D para a idade-índice de 12 anos, ficando somente a região Norte com valor maior para o índice após sete anos (3,13 em 2003 para 3,16 em 2010) (Agnelli, 2015).

O índice CPO-D das regiões menos favorecidas economicamente, como Região Norte e Nordeste, tem apresentado maiores médias em todos os estudos epidemiológicos. Sabe-se que a situação de saúde bucal da população está intimamente relacionada às grandes desigualdades socioeconômicas e ao difícil acesso aos serviços em saúde. Alguns autores consideram a situação socioeconômica como um importante fator de risco para desenvolvimento da cárie dentária (Berti et al., 2013).

\section{Prevalência de cárie dentária na dentição decídua (05 anos):}

Na Tabela 1 é apresentada a média do índice ceo-d nos levantamentos epidemiológicos de 2003 e 2010 para a idade de 5 anos, constatando-se que em 2003 o índice foi de 2,78 com 82,14\% representado pelo componente "c", dentes cariados, ou seja, a cada 100 crianças, mais de 80 possuíam cárie na dentição decídua. Na região Norte a situação é ainda mais desfavorável, pois $89,75 \%$ das crianças com 5 anos apresentavam cárie (Brasil, 2012).

Observa-se redução de 14\% do índice em 2010 assumindo valor para ceo-d de 2,43 (Tabela 1), sendo o componente "c" ainda o mais expressivo correspondendo a $80,2 \%$ do valor total do índice. A prevalência de cárie dentária foi de 53,1\%, ou seja, mais da metade das crianças dessa idade foram acometidas pela doença (Ardenghi et al., 2013).

O estudo de 2010 também avaliou a desigualdade em sua distribuição nas regiões brasileiras e entre crianças com diferentes características sócio demográficas, registrando o maior índice para a prevalência de cárie na região Norte (ceo-d 3,37), ficando com índice cerca de 63\% maior que a região Sudeste (ceo-d 2,07). Outro fato importante e que deve ser reconsiderado é que ao longo dos sete anos mais crianças foram acometidas pela doença na região Norte, pois o percentual de crianças livres de cárie reduziu de 35\% para 34\% comparando 2003 com 2010, conforme a Tabela 1 (Ardenghi et al., 2013).

A relação renda familiar e prevalência de cárie está bem documentada na literatura, onde a relação se estabelece por maior ingestão de alimentos ricos em açúcar, maior dificuldade de acesso aos serviços em saúde e piores padrões de higiene bucal (Ardenghi et al, 2013; Borges et al., 2016).

As desigualdades socioeconômicas e iniquidades na distribuição da cárie dentária no Brasil são responsáveis pela ocorrência desigual da doença em diferentes regiões ou grupos populacionais, em que poucos indivíduos acumulam a maior carga da doença (Cruz \& Narvai, 2018). 
Na tabela abaixo observa-se que somente nas regiões Nordeste e Sudeste houve melhora na condição de saúde bucal com aumento no número de crianças de 5 anos livres de cárie de 2003 para 2010. Segundo Vasconcelos et al. (2018), os dados podem ser justificados pela maior ampliação da cobertura da ESF na região Nordeste e, consequentemente, maior acesso aos serviços em saúde bucal e aumento da cobertura do sistema de abastecimento de água fluoretada na região Sudeste.

Tabela 1. Evolução do índice de cárie dentária e Proporção de crianças livres de cárie dentária para 05 e 12 anos de idade.

\begin{tabular}{|c|c|c|c|c|c|c|}
\hline Ano & Norte & Nordeste & Sudeste & Sul & Centro oeste & Brasil \\
\hline 2003 & 3,22 & 3,21 & 2,50 & 2,62 & 2,67 & 2,78 \\
\hline 2010 & 3,37 & 2,92 & 2,07 & 2,49 & 2,98 & 2,43 \\
\hline Evolução & $+0,15$ & $-0,3$ & $-0,40$ & $-0,13$ & $+0,33$ & $-0,37$ \\
\hline \multicolumn{7}{|c|}{ Proporção de crianças de 5 anos livres de cárie (ceo-d =0) } \\
\hline Ano & Norte & Nordeste & Sudeste & Sul & Centro oeste & Brasil \\
\hline 2003 & $35 \%$ & $35 \%$ & $45 \%$ & $43,3 \%$ & $41,7 \%$ & $40,6 \%$ \\
\hline 2010 & $34 \%$ & $41,6 \%$ & $52 \%$ & $39,4 \%$ & $38,8 \%$ & $46,6 \%$ \\
\hline Evolução & $-1 \%$ & $+6,6 \%$ & $+7 \%$ & $-3,9 \%$ & $-2,9 \%$ & $+6 \%$ \\
\hline \multicolumn{7}{|c|}{ Evolução do índice CPO-D para 12 anos de idade } \\
\hline Ano & Norte & Nordeste & Sudeste & Sul & Centro oeste & Brasil \\
\hline 2003 & 3,13 & 3,19 & 2,30 & 2,31 & 3,16 & 2,78 \\
\hline 2010 & 3,16 & 2,63 & 1,72 & 2,06 & 2,63 & 2,07 \\
\hline Evolução & $+0,03$ & $-0,56$ & $-0,58$ & $-0,25$ & $-0,53$ & $-0,71$ \\
\hline \multicolumn{7}{|c|}{ Proporção de crianças de 12 anos livres de cárie (CPO-D =0) } \\
\hline Ano & Norte & Nordeste & Sudeste & Sul & Centro oeste & Brasil \\
\hline 2003 & $24,1 \%$ & $27,5 \%$ & $37,6 \%$ & $36,7 \%$ & $27,1 \%$ & $31,1 \%$ \\
\hline 2010 & $28 \%$ & $37,7 \%$ & $48,4 \%$ & $39,41 \%$ & $35,6 \%$ & $43,5 \%$ \\
\hline Evolução & $+3,8 \%$ & $+10,2 \%$ & $+10,8 \%$ & $+4,3 \%$ & $+8,5 \%$ & $+12,4 \%$ \\
\hline
\end{tabular}

Fonte: Brasil (2004); Brasil (2012).

A Tabela 2 demonstra que, dentre as capitais da região Norte, Palmas foi a que apresentou menor índice ceo-d com valor de 1,53, ficando abaixo da média nacional 2,43, bem como maior percentual de crianças de 5 anos livres de cárie (53,5\%, maior que a média nacional de 46,6\%). Embora os dados sejam favoráveis, 87,6\% das crianças apresentam dentes cariados. $\mathrm{O}$ predomínio do componente "c", dentes cariados, em detrimento dos dentes obturados demonstra que o acesso ao tratamento odontológico continua sendo um desafio onde a população não consegue um acesso igualitário aos serviços ofertados (Agnelli, 2015).

Santos et al (2016) afirmam que alguns fatores parecem contribuir com esse cenário como a dificuldade de acesso aos serviços odontológicos, hábitos alimentares e de higiene oral inadequadas nos primeiros anos de vida. Os dados referentes ao inquérito realizado em 2010 constituem o primeiro levantamento de saúde bucal de Palmas, capital do Tocantins, com dados representativos para o município. A inexistência de estudos anteriores impossibilita a comparação com os dados apresentados acima (Brasil, 2012). 
Tabela 2: componentes do índice ceo-d para a idade de 5 anos por capital da Região Norte.

\begin{tabular}{|c|c|c|c|c|c|c|c|c|c|}
\hline \multirow[t]{2}{*}{ Componentes } & \multicolumn{2}{|c|}{ Cariado } & \multicolumn{2}{|c|}{ Cariado/Obturado } & \multicolumn{2}{|c|}{ Obturado } & \multicolumn{2}{|c|}{$\begin{array}{l}\text { Perdido por } \\
\text { cárie }\end{array}$} & \multirow{2}{*}{$\begin{array}{l}\text { ceo-d } \\
\text { Média }\end{array}$} \\
\hline & Média & $\%$ & Média & $\%$ & Média & $\%$ & Média & $\%$ & \\
\hline Palmas & 1,34 & 87,6 & 0,01 & $0,7 \%$ & 0,14 & $9,2 \%$ & 0,05 & $3,3 \%$ & 1,53 \\
\hline Belém & 1,93 & 91,6 & 0,03 & 1,4 & 0,10 & 4,7 & 0,04 & 1,9 & 2,14 \\
\hline Macapá & 1,34 & 50,8 & 0,03 & 1,1 & 0,16 & 6,1 & 0,05 & 1,9 & 2,64 \\
\hline Manaus & 2,30 & 79,9 & 0,08 & 2,8 & 0,34 & 11,8 & 0,16 & 5,6 & 2,88 \\
\hline Porto Velho & 2,44 & 84,4 & 0,09 & 3,1 & 0,27 & 9,3 & 0,09 & 3,01 & 2,89 \\
\hline Rio Branco & 2,47 & 82,9 & 0,04 & 1,3 & 0,40 & 13,4 & 0,06 & 2,0 & 2,98 \\
\hline BRASIL & 1,95 & 80,2 & 0,08 & 3,3 & 0,33 & 13,6 & 0,06 & 2,5 & 2,43 \\
\hline
\end{tabular}

Fonte: Brasil (2012).

\section{Prevalência de cárie dentária na dentição permanente (12 anos):}

A idade de 12 anos é estabelecida pela OMS como idade-índice possibilitando comparações do indicador internacionalmente. Como já demonstrado anteriormente, o Brasil passou da condição de "muito alta" prevalência de cárie em escolares em 1980 para "baixa” prevalência em 2010 (Narvai et al., 2006; Agnelli, 2015).

Ao longo dos anos a redução na prevalência de cárie na faixa etária de 12 anos foi bastante significativa. Em 1996 o valor obtido para o índice CPO-D foi 3,1, valor 54\% menor do que o obtido em 1986 (CPO-D 6,7). Em 2003 o valor do índice encontrado foi 2,8, classificado como "média" prevalência de cárie, sendo 59\% menor do que o encontrado no primeiro levantamento, em 1986. A redução da prevalência de cárie entre 2003 e 2010 foi de 25\%, passando de 2,80 para 2,10, com redução do componente “c”, dentes cariados, de 29\% (1,7 em 2003 para 1,2 em 2010) (Brasil, 2012; Agnelli, 2015; Roncalli et al., 2019).

Em 2003 o Brasil atingiu a meta preconizada pela OMS para o ano de 2000 somente para a idade de 12 anos, a qual recomendava um índice CPO-D menor que 3,0, alcançando valor de 2,78. Mesmo os dados sendo favoráveis, o estudo apontou que cerca de $70 \%$ das crianças brasileiras de 12 anos tinham, pelo menos, um dente cariado e as diferenças regionais eram grandes, em especial para as regiões Norte, Nordeste e Centro-Oeste que possuem suas particularidades as quais influenciam na prevalência de cárie dentária (Tobias et al., 2008; Brasil, 2004).

A meta estabelecida pela OMS para 2010 seria índice CPO-D para idade de 12 anos inferior a 1,0 e 90\% das crianças de 5 anos estariam livres de cárie. No entanto, estudos epidemiológicos demonstraram que somente 46,6\% das crianças no ano estavam livres de cárie, ficando longe da meta preconizada pela OMS.

Para 2020, considerando os valores alcançados na pesquisa de 2010, a OMS adaptou as metas para saúde bucal publicadas em um documento intitulado "Global Goals for Oral Health" onde estabelece que 80\% das crianças com 6 anos de idade deverão estar livres de cáries e aos 12 anos o índice CPO-D deverá ser inferior a 1,5 (Vilar et al., 2020). 
Tabela 3: Comparação da meta estabelecida pela OMS e valor alcançado nos levantamentos epidemiológicos no Brasil em 2003 e 2010 para idade de 12 anos.

\begin{tabular}{llllll}
\hline \multicolumn{1}{c}{ Idade } & $\begin{array}{c}\text { Meta da OMS } \\
\text { para 2000 }\end{array}$ & \multicolumn{1}{c}{$\begin{array}{c}\text { SB Brasil } \\
\mathbf{2 0 0 3}\end{array}$} & $\begin{array}{c}\text { Meta da OMS } \\
\text { para 2010 }\end{array}$ & SB Brasil 2010 & $\begin{array}{c}\text { Meta da OMS } \\
\text { para 2020 }\end{array}$ \\
\hline 5 a 6 anos & $50 \%$ livres de cárie & $\begin{array}{l}40,6 \% \text { livres } \\
\text { de cárie }\end{array}$ & $\begin{array}{l}90 \% \text { livres de } \\
\text { cárie }\end{array}$ & $\begin{array}{l}46,6 \% \text { livres de } \\
\text { cárie }\end{array}$ & $\begin{array}{l}80 \% \text { livres de } \\
\text { cárie }\end{array}$ \\
\hline 12 anos & CPO-D $<$ ou $=3,0$ & $\begin{array}{l}\text { CPO-D }= \\
2,78\end{array}$ & $\begin{array}{l}\text { CPO-D }<\text { ou }= \\
1,0\end{array}$ & CPO-D $=2,07$ & CPO-D $<1,5$ \\
& & & & &
\end{tabular}

Fonte: Lucas et al., (2005); Vilar et al. (2020).

Embora observa-se uma redução na prevalência e na gravidade da cárie no país, a diferença nos índices CPO-D entre as regiões também se manteve para a idade de 12 anos ao longo dos anos e a região Norte tem demonstrado os piores resultados comparados às demais regiões. Este fato pode estar associado às condições socioeconômicas e dificuldade no acesso aos serviços odontológicos, bem como a deficiência em ações de promoção e prevenção em saúde bucal nas regiões menos favorecidas (Vasconcelos et al, 2018).

Com relação ao número de crianças livres de cárie, a Tabela 1 demonstra que em todas as regiões houve melhora no percentual para a idade de 12 anos em 2010 comparado ao ano de 2003 . No entanto a região Norte apresentou a menor diferença comparada às demais regiões $(+3,8 \%)$.

Lacerda et al. (2020) afirmam que, mesmo sendo obrigatória por lei a fluoretação do sistema de abastecimento de água no Brasil desde os anos 70, atualmente o país apresenta cobertura por volta de $60 \%$ com desigualdades entre as regiões. No Sul e Sudeste, mais de $70 \%$ das pessoas são beneficiadas pela fluoretação no sistema de abastecimento de água, enquanto na região Norte, menos de $30 \%$.

Embora tal medida de promoção à saúde esteja amparada por lei, ainda não é uma realidade tocantinense, pois dos 139 municípios do estado do Tocantins, apenas 29 possuem água fluoretada, resultando em 110 municípios sem fluoretação. O autor afirma ainda que 9 em cada 10 amostras do estudo não estavam no padrão adequado de menor risco à fluorose dentária e maior proteção contra a cárie dentária, necessitando dessa forma, maior rigor na estratégia de adição do flúor à água de abastecimento (Lacerda et al., 2020).

Comparando os dados aos 5 e 12 anos (Tabela 2), observa-se que a redução de cárie foi maior na dentição permanente do que na dentição decídua, tendo em vista que, para o ceo-d observou-se uma redução de $13,2 \%$, enquanto o percentual na dentição permanente foi maior atingindo $25,5 \%$. Houve aumento em todas as regiões do percentual de crianças livres de cárie aos 12 anos, ao contrário das crianças de 5 anos que houve melhora somente nas regiões Nordeste e Sudeste (Vasconcelos et al., 2018). Uma possível explicação para uma menor redução da cárie dentária na faixa etária de 5 anos poderia ser a baixa taxa de assistência à saúde bucal na primeira infância (Gimenez et.al., 2016)

O estudo de 2010 aponta ainda que a região Norte apresentou maior proporção de indivíduos que nunca foram ao dentista e maior proporção de consultas motivadas pela dor, quando comparadas às regiões Sul e Sudeste (Peres et al., 2012).

A Tabela 4 compara os dados das capitais da região Norte e demonstra que Palmas é a segunda capital com menor índice CPO-D (2,35) para idade de 12 anos, ficando atrás somente de Manaus (2,34) com 57,9\% do índice representado por dentes cariados. O percentual dos dentes obturados em Palmas foi menor na faixa etária de 5 anos, sendo 9,2\% e para a idade de 12 anos foi $30,2 \%$ do índice de dentes obturados.

Este dado demonstra que em Palmas as crianças em idades iniciais apresentam mais dentes cariados que as crianças de 12 anos. Nesse contexto, mais pesquisas precisam ser realizadas no município para determinar as condições de saúde bucal das crianças e adolescentes a fim de conhecer as fragilidades e dificuldades, propondo estratégias efetivas para redução da incidência de cárie na infância e adolescência. 
Tabela 4: componentes do índice CPO-D para a idade de 12 anos por capital da Região Norte

\begin{tabular}{|c|c|c|c|c|c|c|c|c|c|}
\hline \multirow[t]{2}{*}{ Componentes } & \multicolumn{2}{|c|}{ Cariado } & \multicolumn{2}{|c|}{$\begin{array}{c}\text { Obturado com } \\
\text { cárie }\end{array}$} & \multicolumn{2}{|c|}{ Obturado } & \multicolumn{2}{|c|}{ Perdido por cárie } & \multirow{2}{*}{$\begin{array}{l}\text { CPO-D } \\
\text { Média }\end{array}$} \\
\hline & Média & $\%$ & Média & $\%$ & Média & $\%$ & Média & $\%$ & \\
\hline Palmas & 1,36 & 57,9 & 0,07 & 3,0 & 0,71 & 30,2 & 0,22 & 9,4 & 2,35 \\
\hline Belém & 1,89 & 77,1 & 0,10 & 4,1 & 0,30 & 12,2 & 0,16 & 6,5 & 2,45 \\
\hline Macapá & 1,61 & 65,4 & 0,03 & 1,2 & 0,60 & 24,2 & 0,21 & 8,5 & 2,46 \\
\hline Manaus & 1,49 & 63,7 & 0,08 & 3,4 & 0,66 & 28,2 & 0,11 & 4,7 & 2,34 \\
\hline Porto Velho & 2,80 & 67,5 & 0,15 & 3,6 & 0,97 & 23,4 & 0,22 & 5,3 & 4,15 \\
\hline Rio Branco & 1,21 & 46 & 0,25 & 9,5 & 1,03 & 39,2 & 0,14 & 5,3 & 2,63 \\
\hline BRASIL & 1,12 & 54,1 & 0,09 & 4,3 & 0,73 & 35,3 & 0,12 & 5,8 & 2,07 \\
\hline
\end{tabular}

Fonte: Brasil (2012).

Estes dados corroboram com o estudo de Peres et al. (2012), em que afirmam que o uso recente de serviço odontológico, avaliado no estudo de 2010, foi maior entre escolares de 7 a 19 anos, mais pobres ou mais ricos. $\mathrm{O}$ autor afirma ainda que a oferta de serviços de saúde pelo SUS, embora tenha aumentado, é provavelmente maior para jovens em detrimento das crianças, reproduzindo o modelo histórico de organização dos serviços públicos odontológicos no Brasil que priorizou a população em idade escolar.

Huang et al. (2019) descrevem em estudo que, embora metade das famílias tenha relatado levar seus filhos ao dentista, 96\% das crianças ainda apresentavam cárie não tratada. O autor afirma que esses dados podem indicar falta de consciência dos pais e cuidadores sobre a importância da prevenção e tratamento da cárie nos dentes decíduos na infância e a crença comum de que os "dentes de leite" serão substituídos e, por isso, não são importantes. Tais resultados reforçam o dever de orientação por parte dos profissionais de saúde bucal às crianças e seus responsáveis quanto à higienização dos dentes, contribuindo para a melhora da saúde bucal e consequentemente redução da incidência de cárie em crianças na primeira infância (Vilar et al., 2020).

\section{Considerações Finais}

Embora o Brasil seja classificado como "baixa" prevalência de cárie para a idade de 12 anos, conforme os achados do último estudo realizado em 2010, as diferenças regionais permaneceram ao longo dos anos com maior predomínio do componente "c", dentes cariados. Os dados acima apresentados referentes ao município de Palmas no ano de 2010 foram os únicos encontrados na literatura, haja vista a insuficiência de um histórico dos dados do município em estudos anteriores. Portanto, mais estudos epidemiológicos, incluindo área urbana e rural, devem ser realizados no município a fim de conhecer a situação de saúde bucal da população, bem como avaliar programas e subsidiar o planejamento de ações em saúde bucal.

Sugere-se com isso que o município inclua o monitoramento das condições de saúde bucal da população, em especial das crianças e adolescentes por meio de inquéritos epidemiológicos, os quais poderão colaborar na formulação de programas voltados para a primeira infância com foco no Pré-Natal Odontológico, tendo em vista que o período da gestação é mais favorável às mudanças de hábitos, estando as gestantes mais receptivas para novos conhecimentos e hábitos saudáveis. 


\section{Referências}

Antunes, J. L. F. \& Narvai, P. C. (2010). Políticas de saúde bucal no Brasil e seu impacto sobre as desigualdades em saúde. Rev Saúde Pública, 44(2), 360-5.

Agnelli, P. B. (2015). Variação do índice CPOD do Brasil no período de 1980 a 2010. Rev. bras. odontol. 72 (2), 10-5.

Ardenghi, T. M., Piovesan, C. \& Antunes, J. L. F. (2013). Desigualdades na prevalência de cárie dentária não tratada em crianças pré-escolares no Brasil. Rev. Saúde Pública, 47 (Supl 3),129-137.

BRASIL. (2012). Ministério da Saúde. Secretaria de Atenção à Saúde. Secretaria de Vigilância em Saúde. SB Brasil 2010: Pesquisa Nacional de Saúde Bucal: resultados principais / Ministério da Saúde. Secretaria de Atenção à Saúde. Secretaria de Vigilância em Saúde. - Brasília: Ministério da Saúde.

Brasil. (2004). Projeto SB Brasil 2003: condições de saúde bucal da população brasileira 2002-2003: resultados principais / Ministério da Saúde, Secretaria de Atenção à Saúde, Departamento de Atenção Básica. - Brasília: Ministério da Saúde.

Berti, M., Furlanetto, D. L. C, Waker, M. M. S, Baltazar, M. M. M. \& Bianchi, F. J. (2013). Levantamento epidemiológico de cárie dentária em escolares de 5 e 12 anos de idade do município de Cascavel, PR. Cad. saúde colet., 21(4).

Borges, T. S., Schwanke, N. L., Reuter, C. P., Kraether Neto, L. \& Burgos, M. S. (2016). Fatores associados à cárie: pesquisa de estudantes do sul do Brasil. Ver Paul. Pediatr., 34, 489-494.

Cruz, M. G. B \& Narvai, P. C. (2018). Cárie e água fluoretada em dois municípios brasileiros com baixa prevalência da doença. Rev Saúde Pública. 52 (28).

Demeu, A. J. M., Viudes, L. P., Barbosa, L. C. \& Nascimento, V. R. (2019). Prevalência de cárie em crianças do ensino fundamental de Umuarama, Paraná. Arch Health Invest, 8(10):592-596.

Dourado, M. R., Rebelo, J. H. A., Rocha, A. L. \& Santa-Rosa, T. T. A. (2017). Prevalência de cárie em escolares da zona rural de Indaiabira, Minas Gerais, Brasil. Rev. APS. 20(1): 89 - 97

Freire, M. C. M., Reis, S. C. G. B., Figueiredo, N., Peres, K. G., Moreira, R.S. \& Antunes, J. L. F. (2013). Determinantes individuais e contextuais da cárie em crianças brasileiras de 12 anos em 2010. Rev. Saúde Pública, Dec. 47 (supl.3).

Gimenez, T., Bispo, B. A., Souza, D. P., Viganó, M. E., Wanderley, M. T., Mendes, F. M., Bonecker, M. \& Braga, M. M. (2016). Does the Decline in Caries Prevalence of Latin American and Caribbean Children Continue in the New Century? Evidence from Systematic Review with Meta-Analysis. PLOS ONE. 11. 10.1371/journal.pone.0164903.

Huang, D., Sokal-Gutierrez, K. \& Chung, K. (2019). Maternal and Child Nutrition and Oral Health in Urban Vietnam. International Journal of Environmental Research and Public Health, 16, 5-8.

Heozor-Ejiofor, Z., Worthington, H. V., Walsh, T., O'Malley, L., Clarkson, J. E., Macey, R., Alam, R., Tugwell, P., Welch, V. \& Glenny, A. M. Water fluoridation for the prevention of dental caries. Cochrane Database Syst Rev. 2015(6):CD010856. 10.1002/14651858.CD010856.pub2.

Kopycka-Kedzierawski, D. T. \& Billings, R. J. (2011). Prevalência de cárie dentária e utilização de cuidados dentários em crianças pré-escolares urbanas matriculadas em um estudo de eficácia comparativa. Arquivos europeus de odontopediatria: jornal oficial da Academia Europeia de Odontopediatria, 12 (3), 133-138. https://doi.org/10.1007/BF03262794.

Lacerda, A. P. A. G., Oliveira, N. A., Pinheiro, H. H. C., Assis, K. M. L. \& Cury, J. A. (2020). Fluoretação da água dos dez maiores municípios do estado do Tocantins, Brasil. Ciênc. Saúde Coletiva, 25(4).

Lucas, S. D, Portela, M. C. \& Mendonça, L. L. Variações no nível de cárie dentária entre crianças de 5 e 12 anos em Minas Gerais, Brasil. CAD. SAÚDE PÚBLICA $21(1) \cdot$ FEV 2005

Martins, A. M. E. B. L., Melo, F. S., Fernandes, F. M., Sorte, J. A. B., Coimbra, L. G. A. \& Batista, R. C. (2005). Levantamentos epidemiológicos brasileiros das condições de saúde bucal. UNIMONTES CIENTÍFICAS, Montes Claros, .7 (1).

Mehta, A. Trends in dental caries in Indian children for the past 25 years. Indian J Dent Res. 29(3):323-328. 10.4103/ijdr.IJDR_615_17.

Melo, C. B. \& Lima, C. M. A. (2009). Estudo epidemiológico da cárie dentária no brasil, período de 1986 a 2003. Revista Paraense de Medicina, 23(4).

Moura, C., Cavalcanti, A. L. \& Bezerra, P. K. M. (2008). Prevalência de cárie dentária em escolares de 12 anos de idade, Campina Grande, Paraíba, Brasil: enfoque socioeconômico. Rev. odonto ciênc. 23(3):256-262.

Moysés, S. J., Pucca Junior, G. A., Paludetto Junior, M. A. \& Moura, L. (2013). Avanços e desafios à Política de Vigilância à Saúde Bucal no Brasil. Rev Saúde Pública,47(Supl 3):161-7.

Narvai, P.C, Frazão, P. \& Castellanos, R. A. (1999). Declínio na experiência de cárie em dentes permanentes de escolares brasileiros no final do século XX. Rev. Odontologia e Sociedade, 1 (1).

Narvai, P. C., Frazão, P., Roncalli, A. G. \& Antunes, J. L. F. (2006). Cárie dentária no Brasil: declínio, iniquidade e exclusão social. Rev Panam Salud Publica. 19(6):385-93.

Pontigo-Loyola, A. P., Márquez-Corona, M. L., Minaya-Sánchez, M., Lucas-Rincón, S. E., Casanova-Rosado, J. F., Robles-Minaya, J. L., Casanova-Sarmiento, J. A., Casanova-Rosado, A. J., Mendoza-Rodriguez, M. \& Medina-Solís, C. E. (2020). Correlation between the caries status of the first permanent molars and the overall DMFT Index. Medicine (Baltimore). 99 (5): e19061. 
Research, Society and Development, v. 11, n. 2, e31211225667, 2022

(CC BY 4.0) | ISSN 2525-3409 | DOI: http://dx.doi.org/10.33448/rsd-v11i2.25667

Peres, K. G., Peres, M. A., Boing, A. F., Bertoldi, A. D., Bastos, J. L. \& Barros, A. J. D. (2012). Redução das desigualdades sociais na utilização de serviços odontológicos no Brasil entre 1998 e 2008. Rev Saúde Pública, 46(2), 250-8.

Roncalli, A. G., Côrtes, M. I. S. \& Peres, K. G. (2012). Perfis epidemiológicos de saúde bucal no Brasil e os modelos de vigilância. Cad. Saúde Pública, 28 (Sup: S58-S68).

Santos, S. P., Vieira, G. O., Scavuzzi, A. I. F. \& Gomes Filho, I. S. (2016). Práticas alimentares e cárie dentária - uma abordagem sobre a primeira infância. Rev. Assoc. Paul. Cir. Dent. (1).

Silva Junior, M. F., Sousa, M. L. R \& Batista M. J. (2019). Reducing social inequalities in the oral health of an adult population. Braz. Oral Res. 33:102.

Splieth, C. H.; Santamaria, R. M.; Basner, R; Schüler, E. \& Schmoeckel, J. 40-Year Longitudinal Caries Development in German Adolescents in the Light of New Caries Measures. Caries Res. 2019;53(6):609-616. 10.1159/000501263.

Tobias, R., Parente, R. C. P. \& Rebelo, M. A. B. (2008). Prevalência e gravidade da cárie dentária e necessidade de tratamento em crianças de 12 anos de município de pequeno porte inserido no contexto amazônico. Rev Bras Epidemiol. 11(4), 608-618.

Vasconcelos, F. G. G., Gondim, B. L. C., Rodrigues, L. V., Lima-NetO, E. A., \& Valença, A. M. G. (2018). Evolução dos índices ceo-d/cpo-d e de cuidados odontológicos em crianças e adolescentes com base no SB BRASIL 2003 e SB BRASIL 2010. Revista Brasileira De Ciências Da Saúde, 22 (4), 333 - 340.

Vilar, M. O., Pinheiro, W. R. \& Araújo, I. S. (2020). Prevalência de cárie dentária em crianças em condição de vulnerabilidade social. Id on Line Rev.Mult. Psic., 14 (49), 577-586.

Xiao, J., Alkhersa, N., Kopycka-Kedzierawskia, D. T., Billingsa, R. J., Wu, T. T., Castillo, D. A., Rasubala, L., Mal,mstrom, H., Ren, Y. \& Eliav, E. (2019). Prenatal Oral Health Care and Early Childhood Caries Prevention: A Systematic Review and Meta-Analysis. Caries Res., 53:411-421.

Watt, R., Mathur, M. R., Ainda, J., Bonecker, M., Venturelli, R. \& Gansky, S. A. (2018). Oral Health Disparities in Children: a Canary in the Coalmine? Pediatric Clinics of North America, 65, 965-979. 